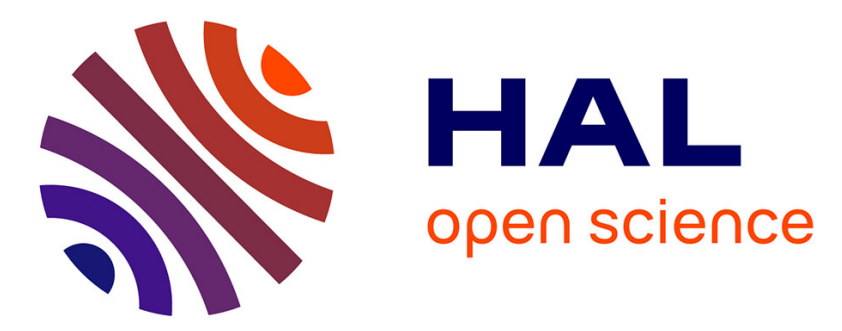

\title{
Bi-Hamiltonian ordinary differential equations with matrix variables
}

\author{
Alexander Odesskii, Vladimir Roubtsov, Vladimir Sokolov
}

\section{To cite this version:}

Alexander Odesskii, Vladimir Roubtsov, Vladimir Sokolov. Bi-Hamiltonian ordinary differential equations with matrix variables. Theoretical and Mathematical Physics, 2012, 171 (1), pp.442 - 447. 10.1007/s11232-012-0043-4 . hal-03054050

\section{HAL Id: hal-03054050 \\ https://univ-angers.hal.science/hal-03054050}

Submitted on 11 Dec 2020

HAL is a multi-disciplinary open access archive for the deposit and dissemination of scientific research documents, whether they are published or not. The documents may come from teaching and research institutions in France or abroad, or from public or private research centers.
L'archive ouverte pluridisciplinaire HAL, est destinée au dépôt et à la diffusion de documents scientifiques de niveau recherche, publiés ou non, émanant des établissements d'enseignement et de recherche français ou étrangers, des laboratoires publics ou privés. 
Theoretical and Mathematical Physics, 171(1): 442-447 (2012)

\section{BI-HAMILTONIAN ORDINARY DIFFERENTIAL EQUATIONS WITH MATRIX VARIABLES}

(C) A. V. Odesskii, ${ }^{*}$ V. N. Rubtsov ${ }^{\dagger}$ and V. V. Sokolov ${ }^{\ddagger}$

We consider a special class of Poisson brackets related to systems of ordinary differential equations with matrix variables. We investigate general properties of such brackets, present an example of a compatible pair of quadratic and linear brackets, and find the corresponding hierarchy of integrable models, which generalizes the two-component Manakov matrix system to the case of an arbitrary number of matrices.

Keywords: integrable ordinary differential equation with matrix unknowns, bi-Hamiltonian formalism, Manakov model

\section{Introduction}

We consider systems of ordinary differential equations (ODEs) of the form

$$
\frac{d x_{\alpha}}{d t}=F_{\alpha}(\mathbf{x}), \quad \mathbf{x}=\left(x_{1}, \ldots, x_{N}\right),
$$

where $x_{i}$ are $m \times m$ matrices and $F_{\alpha}$ are (noncommutative) polynomials. There exist systems (1) that are integrable for any $m$. For example, the system

$$
u_{t}=u^{2} v-v u^{2}, \quad v_{t}=0
$$

is integrable by the inverse scattering method for matrices $u$ and $v$ of any size $m$. If $u$ is a matrix such that $u^{T}=-u$ and $v$ is a constant diagonal matrix, then (2) is equivalent to the $m$-dimensional Euler top. The integrability of this model was established by Manakov [1].

Here, we construct an integrable generalization of system (2) to the case of arbitrary $N$ using the biHamiltonian approach [2]. This approach is based on the notion of a pair of compatible Poisson brackets. Two Poisson brackets $\{\cdot, \cdot\}_{1}$ and $\{\cdot, \cdot\}_{2}$ are said to be compatible if

$$
\{\cdot, \cdot\}_{\lambda}=\{\cdot, \cdot\}_{1}+\lambda\{\cdot, \cdot\}_{2}
$$

is a Poisson bracket for any $\lambda$.

If bracket (3) is degenerate for any $\lambda$, then a hierarchy of integrable Hamiltonian ODE systems can be constructed using the following assertion.

*Brock University, St. Catharines, Canada, e-mail: aodesski@brocku.ca.

${ }^{\dagger}$ Institute for Theoretical and Experimental Physics, Moscow, Russia; LAREMA, CNRS, Université d'Angers, Angers, France, e-mail: volodya@univ-angers.fr.

${ }^{\ddagger}$ Landau Institute for Theoretical Physics, RAS, Moscow, Russia, e-mail: sokolov@itp.ac.ru.

Prepared from an English manuscript submitted by the authors; for the Russian version, see Teoreticheskaya $i$ Matematicheskaya Fizika, Vol. 171, No. 1, pp. 26-32, April, 2012. Original article submitted May 7, 2011. 
Theorem 1 [3], [4]. Let

$$
C(\lambda)=C_{0}+\lambda C_{1}+\lambda^{2} C_{2}+\ldots, \quad \bar{C}(\lambda)=\bar{C}_{0}+\lambda \bar{C}_{1}+\lambda^{2} \bar{C}_{2}+\cdots
$$

be the Taylor expansions of any two Casimir functions for the bracket $\{\cdot, \cdot\}_{\lambda}$. Then the coefficients $C_{i}$ and $\bar{C}_{j}$ pairwise commute with respect to both the brackets $\{\cdot, \cdot\}_{1}$ and $\{\cdot, \cdot\}_{2}$.

In the opposite case where the bracket $\{\cdot, \cdot\}_{1}$ is nondegenerate, for example, there is another way to construct an integrable hierarchy. The ratio $R=\Pi_{2} \Pi_{1}^{-1}$, where $\Pi_{i}$ is the Poisson tensor for $\{\cdot, \cdot\}_{i}$, defines a so-called recursion operator whose spectrum provides the set of functions in involution with respect to both brackets. In this case, the formula $\Pi_{k}=R^{k} \Pi_{1}$ yields an infinite sequence of pairwise compatible Poisson brackets.

For an important class of Poisson brackets related to systems (1), the corresponding Hamiltonian operator can be expressed in terms of left and right multiplication operators given by polynomials in $x_{1}, \ldots, x_{N}[5]$. Such brackets have the following two properties:

1. They are $G L_{m}$-adjoint invariant.

2. The bracket between the traces of any two matrix polynomials $P_{i}\left(x_{1}, \ldots, x_{N}\right), i=1,2$, is a trace of some other matrix polynomial $P_{3}$.

Such brackets have been called non-Abelian Poisson brackets.

We here consider compatible pairs of non-Abelian Poisson brackets where $\{\cdot, \cdot\}_{1}$ is linear and $\{\cdot, \cdot\}_{2}$ is quadratic.

\section{Non-Abelian Poisson brackets}

We consider Poisson brackets of the forms

$$
\begin{aligned}
& \left\{x_{i, \alpha}^{j}, x_{i^{\prime}, \beta}^{j^{\prime}}\right\}_{1}=b_{\alpha, \beta}^{\gamma} x_{i, \gamma}^{j^{\prime}} \delta_{i^{\prime}}^{j}-b_{\beta, \alpha}^{\gamma} x_{i^{\prime}, \gamma}^{j} \delta_{i}^{j^{\prime}} \\
& \left\{x_{i, \alpha}^{j}, x_{i^{\prime}, \beta}^{j^{\prime}}\right\}_{2}=r_{\alpha \beta}^{\gamma \epsilon} x_{i, \gamma}^{j^{\prime}} x_{i^{\prime}, \epsilon}^{j}+a_{\alpha \beta}^{\gamma \epsilon} x_{i, \gamma}^{k} x_{k, \epsilon}^{j^{\prime}} \delta_{i^{\prime}}^{j}-a_{\beta \alpha}^{\gamma \epsilon} x_{i^{\prime}, \gamma}^{k} x_{k, \epsilon}^{j} \delta_{i}^{j^{\prime}},
\end{aligned}
$$

where $x_{i, \alpha}^{j}$ are elements of the matrix $x_{\alpha}$ and $\delta_{i}^{j}$ is the Kronecker delta. Summation over repeated indices is assumed. Here and hereafter, we use Latin indices ranging from 1 to $m$ for the matrix elements and Greek indices ranging from 1 to $N$ to label the matrices.

Theorem 2. Brackets of forms (4) and (5) are both invariant under the $G L_{m}$-action $x_{\alpha} \rightarrow u x_{\alpha} u^{-1}$, where $u \in G L_{m}$. Moreover, these brackets have the property that the bracket between traces of any two matrix polynomials is a trace of a matrix polynomial. Any linear or quadratic Poisson bracket with these two properties has the respective form (4) or (5).

There are many publications devoted to quadratic Poisson brackets appearing in the classical version of the inverse scattering method [6], but these brackets do not have the properties in Theorem 2 .

Theorem 3. 1. Formula (4) defines a Poisson bracket iff

$$
b_{\alpha \beta}^{\mu} b_{\mu \gamma}^{\sigma}=b_{\alpha \mu}^{\sigma} b_{\beta \gamma}^{\mu} .
$$

2. Formula (5) defines a Poisson bracket iff

$$
\begin{aligned}
& r_{\alpha \beta}^{\sigma \epsilon}=-r_{\beta \alpha}^{\epsilon \sigma}, \quad r_{\alpha \beta}^{\lambda \sigma} r_{\sigma \tau}^{\mu \nu}+r_{\beta \tau}^{\mu \sigma} r_{\sigma \alpha}^{\nu \lambda}+r_{\tau \alpha}^{\nu \sigma} r_{\sigma \beta}^{\lambda \mu}=0, \\
& a_{\alpha \beta}^{\sigma \lambda} a_{\tau \sigma}^{\mu \nu}=a_{\tau \alpha}^{\mu \sigma} a_{\sigma \beta}^{\nu \lambda}, \quad a_{\alpha \beta}^{\sigma \lambda} a_{\sigma \tau}^{\mu \nu}=a_{\alpha \beta}^{\mu \sigma} r_{\tau \sigma}^{\lambda \nu}+a_{\alpha \sigma}^{\mu \nu} r_{\beta \tau}^{\sigma \lambda}, \quad a_{\alpha \beta}^{\lambda \sigma} a_{\tau \sigma}^{\mu \nu}=a_{\alpha \beta}^{\sigma \nu} r_{\sigma \tau}^{\lambda \mu}+a_{\sigma \beta}^{\mu \nu} r_{\tau \alpha}^{\sigma \lambda} .
\end{aligned}
$$


Formula (6) means that $b_{\alpha \beta}^{\sigma}$ are structure constants of an associative algebra. Similar Poisson brackets were considered in [7], [8].

We consider quadratic Poisson brackets (5). The tensors $r$ and $a$ transform standardly under the change of basis $x_{\alpha} \rightarrow g_{\alpha}^{\beta} x_{\beta}$ :

$$
r_{\alpha \beta}^{\gamma \sigma} \rightarrow g_{\alpha}^{\lambda} g_{\beta}^{\mu} h_{\nu}^{\gamma} h_{\epsilon}^{\sigma} r_{\lambda \mu}^{\nu \epsilon}, \quad a_{\alpha \beta}^{\gamma \sigma} \rightarrow g_{\alpha}^{\lambda} g_{\beta}^{\mu} h_{\nu}^{\gamma} h_{\epsilon}^{\sigma} a_{\lambda \mu}^{\nu \epsilon},
$$

where $g_{\alpha}^{\beta} h_{\beta}^{\gamma}=\delta_{\alpha}^{\gamma}$.

System of identities (7), (8) admits the discrete involution

$$
r_{\alpha \beta}^{\gamma \sigma} \rightarrow r_{\beta \alpha}^{\sigma \gamma}, \quad a_{\alpha \beta}^{\gamma \sigma} \rightarrow a_{\beta \alpha}^{\sigma \gamma} .
$$

This involution corresponds to the matrix transposition $x_{\alpha} \rightarrow x_{\alpha}^{\mathrm{T}}$. Two brackets related by transformations (9), (10) are said to be equivalent.

There is one more discrete involution:

$$
r_{\alpha \beta}^{\gamma \sigma} \rightarrow r_{\gamma \sigma}^{\alpha \beta}, \quad a_{\alpha \beta}^{\gamma \sigma} \rightarrow a_{\gamma \sigma}^{\alpha \beta} .
$$

Brackets related by (11) can have completely different properties (e.g., different numbers of Casimir functions).

Let $V$ be a vector space with a basis $v_{\alpha}, \alpha=1, \ldots, N$. We define linear operators $r$ and $a$ on the space $V \otimes V$ by

$$
r v_{\alpha} \otimes v_{\beta}=r_{\alpha \beta}^{\sigma \epsilon} v_{\sigma} \otimes v_{\epsilon}, \quad a v_{\alpha} \otimes v_{\beta}=a_{\alpha \beta}^{\sigma \epsilon} v_{\sigma} \otimes v_{\epsilon} .
$$

Identities (7) and (8) can then be rewritten in the form

$$
\begin{aligned}
& r^{12}=-r^{21}, \quad r^{23} r^{12}+r^{31} r^{23}+r^{12} r^{31}=0 \\
& a^{12} a^{31}=a^{31} a^{12}, \quad \sigma^{23} a^{13} a^{12}=a^{12} r^{23}-r^{23} a^{12}, \quad a^{32} a^{12}=r^{13} a^{12}-a^{32} r^{13}
\end{aligned}
$$

Here, all operators act in $V \otimes V \otimes V, \sigma^{i j}$ denotes the transposition of the $i$ th and $j$ th components of the tensor product, and $a^{i j}$ and $r^{i j}$ denote the operators $a$ and $r$ acting in the product of the $i$ th and $j$ th components.

Involution (11) corresponds to $a \rightarrow a^{*}$ and $r \rightarrow r^{*}$, where $a^{*}$ and $r^{*}$ act in the dual space $V^{*}$; involution (10) corresponds to $a \rightarrow \sigma a \sigma$ and $r \rightarrow \sigma r$, where $\sigma$ acts by permuting the vector spaces in $V \otimes V$. Equivalence transformation (9) corresponds to $a \rightarrow G a G^{-1}$ and $r \rightarrow G r G^{-1}$, where $G=g \otimes g$ and $g \in G L(V)$.

There is a subclass of brackets (5) corresponding to the case where the tensor a equals zero. Relations (7) mean that $r$ is a constant solution of the associative Yang-Baxter equation [9], [10]. Such tensors $r$ can be constructed algebraically as follows.

An anti-Frobenius algebra is an associative algebra $\mathcal{A}$ (not necessarily with unity) with a nondegenerate antisymmetric bilinear form $(\cdot, \cdot)$ satisfying the relation

$$
(x, y z)+(y, z x)+(z, x y)=0
$$

for all $x, y, z \in \mathcal{A}$. In other words, the form $(\cdot, \cdot)$ defines a cyclic cocycle on $\mathcal{A}$.

Theorem 4. There exists a one-to-one correspondence between solutions of (7) up to an equivalence and exact representations of anti-Frobenius algebras up to an isomorphism. 
Proof. The tensor $r$ can be written as $r_{\alpha \beta}^{\gamma \sigma}=\sum_{i, j=1}^{p} g^{i j} y_{\alpha, i}^{\gamma} y_{\beta, j}^{\sigma}$, where $g^{i j}=-g^{j i}$, the matrix $G=\left(g^{i j}\right)$ is nondegenerate, and $p$ is the least possible. Substituting this representation in (7), we find that there exists a tensor $\phi_{i j}^{k}$ such that $y_{\gamma, i}^{\alpha} y_{\beta, j}^{\gamma}=\phi_{i j}^{k} y_{\beta, k}^{\alpha}$. Let $\mathcal{A}$ be the associative algebra with the basis $y_{1}, \ldots, y_{p}$ and the product $y_{i} y_{j}=\phi_{i j}^{k} y_{k}$. We define the antisymmetric bilinear form by $\left(y_{i}, y_{j}\right)=g_{i j}$, where $\left(g_{i j}\right)=G^{-1}$. Then (7) is equivalent to the anti-Frobenius property.

Example 1 (cf. [11]). Let $\mathcal{A}$ be the associative algebra of $N \times N$ matrices with a zero $N$ th row. For a generic element $l$ of $\mathcal{A}^{*}$, the bilinear form $(x, y)=l([x, y])$ is nondegenerate and antisymmetric and satisfies (12). It can be written as $(x, y)=\operatorname{tr}\left([x, y] k^{\mathrm{T}}\right)$, where $k \in \mathcal{A}$. We choose $k_{\alpha \beta}=0$ for $\alpha \neq \beta$, $k_{\alpha \alpha}=\mu_{\alpha}$, and $k_{\alpha N}=1$, where $\alpha, \beta=1, \ldots, N-1$. The corresponding solutions of Eqs. (7) are

$$
\begin{aligned}
& r_{N \alpha}^{\alpha \alpha}=-r_{\alpha N}^{\alpha \alpha}=1, \quad r_{\alpha \beta}^{\alpha \beta}=r_{\alpha \beta}^{\beta \alpha}=r_{\beta \alpha}^{\alpha \alpha}=-r_{\alpha \beta}^{\alpha \alpha}=\frac{1}{\mu_{\alpha}-\mu_{\beta}} \\
& \alpha \neq \beta, \quad \alpha, \beta=1, \ldots, N-1 .
\end{aligned}
$$

The remaining elements of the tensor $r$ and all elements of the tensor $a$ are assumed to be zero. We note that this tensor $r$ is antisymmetric under involution (10). It is equivalent to the tensor

$$
r_{\alpha \beta}^{\alpha \beta}=r_{\alpha \beta}^{\beta \alpha}=r_{\beta \alpha}^{\alpha \alpha}=-r_{\alpha \beta}^{\alpha \alpha}=\frac{1}{\lambda_{\alpha}-\lambda_{\beta}}, \quad \alpha \neq \beta, \quad \alpha, \beta=1, \ldots, N,
$$

where $\lambda_{1}, \ldots, \lambda_{N}$ are arbitrary pairwise distinct parameters. Formula (5) with a zero tensor $a$ defines the corresponding Poisson bracket for the elements of matrices $x_{1}, \ldots, x_{N}$ of arbitrary size $m$. For $m=1$, we have the scalar Poisson bracket

$$
\left\{x_{\alpha}, x_{\beta}\right\}=\frac{\left(x_{\alpha}-x_{\beta}\right)^{2}}{\lambda_{\beta}-\lambda_{\alpha}}, \quad \alpha \neq \beta, \quad \alpha, \beta=1, \ldots, N
$$

If $N$ is even, then Poisson structure (13) is nondegenerate, i.e., the rank of the Poisson tensor $\Pi$ is equal to $N m^{2}$. In the odd case, $\operatorname{rank} \Pi=(N-1) m^{2}$.

It can be verified that the conditions for the compatibility of linear and quadratic brackets (4) and (5) are given by

$$
\begin{aligned}
& b_{\alpha \gamma}^{s} a_{s \beta}^{v u}-b_{\gamma \beta}^{s} a_{\alpha s}^{v u}+b_{s \beta}^{u} a_{\alpha \gamma}^{v s}-b_{\alpha s}^{v} a_{\gamma \beta}^{s u}=0, \\
& b_{\beta \alpha}^{s} r_{s \gamma}^{u v}-b_{\beta s}^{u} r_{\alpha \gamma}^{s v}-b_{s \alpha}^{v} r_{\beta \gamma}^{u s}-b_{\gamma s}^{v} a_{\beta \alpha}^{u s}+b_{s \gamma}^{u} a_{\beta \alpha}^{s v}=0 .
\end{aligned}
$$

There is the following way (the so-called argument shift method) to construct a linear Poisson bracket compatible with a quadratic bracket. A vector $\kappa=\left(\kappa_{1}, \ldots, \kappa_{m}\right)$ is said to be admissible if for any $\alpha$ and $\beta$,

$$
\left(a_{\alpha \beta}^{\sigma \epsilon}-a_{\beta \alpha}^{\epsilon \sigma}+r_{\alpha \beta}^{\sigma \epsilon}\right) \kappa_{\sigma} \kappa_{\epsilon}=0 .
$$

For any admissible vector, the argument shift $x_{\alpha} \rightarrow x_{\alpha}+\kappa_{\alpha} \mathbf{1}$ yields linear Poisson bracket (4) with

$$
b_{\alpha \beta}^{\sigma}=\left(a_{\alpha \beta}^{\epsilon \sigma}+a_{\alpha \beta}^{\sigma \epsilon}+r_{\alpha \beta}^{\sigma \epsilon}\right) \kappa_{\epsilon}
$$

compatible with the quadratic bracket. In Example 1, any admissible vector is proportional to $(1,1, \ldots, 1)$, and the corresponding linear bracket is trivial. 
Example 2. Applying involution (11) to (13), we obtain one more example with a zero tensor $a$ :

$$
r_{\alpha \beta}^{\alpha \beta}=r_{\beta \alpha}^{\alpha \beta}=r_{\alpha \alpha}^{\beta \alpha}=-r_{\alpha \alpha}^{\alpha \beta}=\frac{1}{\lambda_{\alpha}-\lambda_{\beta}}, \quad \alpha \neq \beta, \quad \alpha, \beta=1, \ldots, N .
$$

It is easy to verify that any vector $\left(\kappa_{1}, \ldots, \kappa_{N}\right)$ is admissible in this case. All elements of the matrix $\sum_{\alpha=1}^{N} x_{\alpha}$ are Casimir functions for both quadratic Poisson bracket $\{\cdot, \cdot\}_{2}$ given by (15) and for the corresponding linear bracket $\{\cdot, \cdot\}_{1}$. We can therefore fix

$$
\sum_{\alpha=1}^{N} x_{\alpha}=C
$$

where $C$ is a constant matrix. Hamiltonians of the hierarchy commuting with respect to both $\{\cdot, \cdot\}_{2}$ and $\{\cdot, \cdot\}_{1}$ are given by

$$
\operatorname{tr} x_{\alpha}^{k}, \quad \operatorname{tr} x_{\alpha}^{k} \sum_{\beta \neq \alpha} \frac{x_{\beta}}{\lambda_{\alpha}-\lambda_{\beta}}, \quad k=1,2, \ldots
$$

The Casimir functions of $\{\cdot, \cdot\}_{1}$ belong to this set, which allows obtaining the entire hierarchy constructively. The linear Casimir functions for $\{\cdot, \cdot\}_{1}$ are $\operatorname{tr} x_{\alpha}$, where $\alpha=1, \ldots, N$. The dynamical system corresponding to the simplest Hamiltonian $\operatorname{tr} x_{N}$ and the Poisson structure $\{\cdot, \cdot\}_{2}$ has the form

$$
\frac{d x_{\alpha}}{d t}=\frac{x_{N} x_{\alpha}-x_{\alpha} x_{N}}{\lambda_{N}-\lambda_{\alpha}}, \quad \alpha=1, \ldots, N-1
$$

There exists the quadratic Casimir function of the linear bracket

$$
H=\frac{1}{2} \sum_{\alpha=1}^{N} \frac{1}{c_{\alpha}} \operatorname{tr} x_{\alpha}^{2}
$$

where $c_{\alpha}$ are arbitrary constants. The system corresponding to this Hamiltonian and the Poisson bracket $\{\cdot, \cdot\}_{2}$ is given by

$$
\frac{d x_{\alpha}}{d t}=\sum_{\beta \neq \alpha} \frac{x_{\alpha} x_{\beta}^{2}-x_{\beta}^{2} x_{\alpha}}{\left(\lambda_{\alpha}-\lambda_{\beta}\right) c_{\beta}}+\sum_{\beta \neq \alpha} \frac{x_{\beta} x_{\alpha}^{2}-x_{\alpha}^{2} x_{\beta}}{\left(\lambda_{\alpha}-\lambda_{\beta}\right) c_{\alpha}} .
$$

This system can be written in the bi-Hamiltonian form

$$
\frac{d \mathbf{x}}{d t}=\{\mathbf{x}, \operatorname{grad}(\operatorname{tr} H)\}_{2}=\{\mathbf{x}, \operatorname{grad}(\operatorname{tr} K)\}_{1},
$$

where

$$
K=\frac{1}{3} \sum_{\alpha=1}^{N} \frac{1}{c_{\alpha}^{2}} \operatorname{tr} x_{\alpha}^{3}
$$

If $N=2$, then system (16) is equivalent to (2).

\section{Conclusion}

We have constructed some examples of linear and quadratic Poisson brackets naturally related to matrix ODEs. Similar Poisson, symplectic, and many other interesting algebro-geometric structures were recently considered in a series of works developing the ideas of M. Kontsevich's approach to constructing noncommutative symplectic geometry [12]. In this connection, we mention the books and papers of 
P. Etingof, V. Ginzburg, W. Crawley-Boevey, L. Le Bryun, M. Van den Bergh, and many others devoted to Calogero-Moser spaces, the symplectic and Poisson geometry of quiver path algebras, necklace and double Poisson structures, Leibniz-Loday algebras, Rota-Baxter operators and their generalizations, etc.

In our next paper (currently being finished), we will establish the role and place of our non-Abelian structures in the framework of the (non)commutative algebro-geometric notions listed above. We will discuss the relations of our quadratic Poisson structures to the Poisson geometry of the affine varieties that are the coordinate rings of representation spaces (modulo the adjoint $G L$-action), describe the classification of the quadratic brackets for free associative algebras and its relation to the classification of "quadratic" double Poisson structures. We will describe the symplectic foliations and the Casimir functions of our brackets and also their relation to noncommutative integrable systems.

Another interesting problem of great importance is to construct a quantization of the non-Abelian brackets and the corresponding integrable equations and to clarify their relations to the dynamical YangBaxter equation and its different versions. These issues are not discussed in the present paper, but we hope to consider them elsewhere.

Acknowledgments. The authors are grateful to A. Alekseev, J. Avan, E. B. Vinberg, M. Kontsevich, A. Polischchuk, and M. Semenov-Tian-Shansky for the useful discussions and to Y. Kordyukov for the helpful remarks. One of the authors (V. V. S.) is grateful to Angers University, Brock University, and Dijon University for the hospitality while the paper was written.

This work was supported in part by the MATPYL program ("Non-commutative integrable systems," V. N. R. and V. V. S.), the ANR "DIADEMS" project (support of visits in Angers, V. V. S.), the Russian Foundation for Basic Research (Grant No. 11-01-00341-a; V. N. R. and V. V. S.), and the Russian Federal Agency for Science and Innovation (Grant No. 14.740.11.0347).

\section{REFERENCES}

1. S. V. Manakov, Funct. Anal. Appl., 10, No. 4, 328-329 (1976).

2. F. Magri, J. Math. Phys., 19, 1156-1162 (1978).

3. F. Magri, P. Casati, G. Falqui, and M. Pedroni, "Eight lectures on integrable systems," in: Integrability of Nonlinear Systems (Lect. Notes Phys., Vol. 495, Y. Kosmann-Schwarzbach, K. M. Tamizhmani, and B. Grammaticos, eds.), Springer, Heidelberg (1997), p. 256-296.

4. I. M. Gelfand and I. Zakharevich, Selecta Math., n.s., 6, 131-183 (2000).

5. A. V. Mikhailov and V. V. Sokolov, Comm. Math. Phys., 211, 231-251 (2000).

6. A. G. Reyman and M. A. Semenov-Tian-Shansky, Phys. Lett. A, 130, 456-460 (1988).

7. A. V. Odesskii and V. V. Sokolov, J. Phys. A, 39, 12447-12456 (2006).

8. A. Pichereau and G. Van de Weyer, J. Algebra, 319, 2166-2208 (2008).

9. M. Aguiar, J. Algebra, 244, 492-532 (2001).

10. T. Schedler, Math. Res. Lett., 10, 301-321 (2003).

11. A. G. Élashvili, Funct. Anal. Appl., 16, No. 4, 326-328 (1982).

12. M. Kontsevich, "Formal (non)commutative symplectic geometry," in: The Gelfand Mathematical Seminars, 1990-1992 (L. Corwin, I. Gelfand, and J. Lepowsky, eds.), Birkhäuser, Boston, Mass. (1993), pp. 173-187. 